\title{
Burkholdines 1097 and 1229, Potent Antifungal Peptides from Burkholderia ambifaria $2.2 \mathrm{~N}$
}

2010

Vol. 12, No. 4

$664-666$

\author{
Kamilia A. Tawfik, ${ }^{\dagger, \ddagger}$ Peter Jeffs, ${ }^{\S}$ Brian Bray," George Dubay, ${ }^{\perp}$ \\ Joseph O. Falkinham III, ${ }^{\#}$ Mostafa Mesbah, ${ }^{\dagger}$ Diaa Youssef, ${ }^{\dagger}$ \\ Sherief Khalifa, ${ }^{\nabla}$ and Eric W. Schmidt ${ }^{\star, \neq}$
}

Department of Pharmacognosy, Suez Canal University, Ismailia, Egypt 41522,

Department of Medicinal Chemistry, University of Utah, Salt Lake City, Utah 84112,

President SciClin Associates, Chapel Hill, North Carolina 27514, Senior Vice President,

Kainos Medicine, Morrisville North Carolina 27560, Department of Chemistry, Duke

University, Durham North Carolina 27708, Department of Biological Sciences, Virginia

Polytechnic Institute and State University, Blacksburg, Virginia 24061, and Faculty of

Pharmacy, Misr International University, Cairo, Egypt 11341

ewsl@utah.edu

Received November 14, 2009

\section{ABSTRACT}

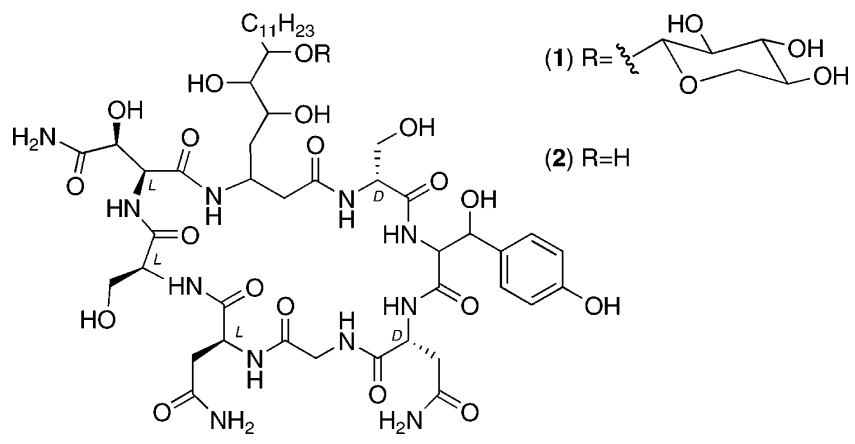

Potent antifungal cyclic lipopeptides, burkholdines (Bk), were isolated from a culture of Burkholderia ambifaria 2.2N. Bk-1229 (1) and Bk-1097 (2) are octapeptides comprised of nonproteinogenic amino acids, including $\beta$-hydroxytyrosine, $\beta$-hydroxyasparagine, and a new fatty acyl amino acid. 1 and 2 are fungicidal against a panel of fungi with potencies $2-60$-fold better than amphotericin $B$ control.

Fungal infections of agricultural plants continue to impact crop growth and yield. While the contributions of synthetic pesticides and herbicides have proven to be of utmost value, the toxicity and slow degradability of many of these compounds in the environment are a continuing problem. The antifungal activities exhibited by bacteria, such as

\footnotetext{
Suez Canal University.

$\doteqdot$ University of Utah.

\& President SciClin Associates.

"Senior Vice President, Kainos Medicine.

${ }^{\perp}$ Duke University.

\# Virginia Polytechnic Institute and State University.

$\nabla$ Misr International University.
}

Burkholderia and Pseudomonas, have led to a strong interest in exploiting these properties as biological agents to control fungal infections. ${ }^{1-3}$ In an effort to discover a biological treatment for these infections, Burkholderia ambifaria $2.2 \mathrm{~N}$ was identified as a potent lead. ${ }^{4,5}$ Bioassay-guided purifica-

(1) Casida, L. E., Jr. U.S. Patent 5, 232, 850, 1993.

(2) Casida, L. E., Jr. Minireviews: Microb. Ecol. 1998, 15, 1-8.

(3) Gross, H.; Loper, J. E. Nat. Prod. Rep. 2009, 26, 1408-1446.

(4) Cain, C.; Henry, A. T.; Walado, R. H., III; Casida, L. J., Jr.; Falkinham, J. O., III Appl. Environ. Microbiol. 2000, 66, 4139-4141.

(5) Coenye, T.; Mahenthiralingam, E.; Henry, D.; LiPuma, J. J.; Laevens, S.; Gillis, M.; Speert, D. P.; Vandamme, P. Int. J. Syst. Evol. Microbiol. 2001, 51, 1481-1490. 
tion of the active principles from $B$. ambifaria extracts using a panel of fungi including Saccharomyces cerevisiae, Candida albicans, and Aspergillus niger led to the discovery of a complex mixture of lipopeptides, the burkholdines. Here, we report the structure elucidation of Bk-1229 (1) and Bk1097 (2), which exhibit potent antifungal activity.

Sequential solvent extraction of spray-dried B. ambifaria 2.2N cells with dichloromethane, 2-propanol, and 2-propanol: $\mathrm{H}_{2} \mathrm{O}$ led to recovery of fractions with increasing antifungal activity. Fractions (250 mg) were further purified on an open $\mathrm{C}_{18}$ column, followed by reversed-phase HPLC using a T3Atlantis column (Waters), to yield mixtures of $\mathbf{1}$ and Bk$1213(900 \mu \mathrm{g})$ and 2 and Bk-1081 (800 $\mu \mathrm{g})$. High-resolution mass spectral analysis revealed ions at $\mathrm{m} / \mathrm{z} 1230.5746$ $[\mathrm{M}+\mathrm{H}]^{+}$and $1252.5569[\mathrm{M}+\mathrm{Na}]^{+}$for $\mathbf{1}\left(\mathrm{C}_{52} \mathrm{H}_{83} \mathrm{~N}_{11} \mathrm{O}_{23}\right)$ and $\mathrm{m} / \mathrm{z}$ 1098.5321 $[\mathrm{M}+\mathrm{H}]^{+}$and $1120.5154[\mathrm{M}+\mathrm{Na}]^{+}$for 2 $\left(\mathrm{C}_{47} \mathrm{H}_{75} \mathrm{~N}_{11} \mathrm{O}_{19}\right)$, while Bk-1213 and Bk-1081 were lighter by a single oxygen than $\mathbf{1}$ and $\mathbf{2}$, respectively, indicating that these are previously unreported compounds. 1 differs from 2 by $\mathrm{C}_{5} \mathrm{H}_{8} \mathrm{O}_{4}$, which has been assigned as xylose. Further purification of the mixtures resulted in essentially pure $1(400 \mu \mathrm{g})$ and $2(300 \mu \mathrm{g})$.

Using 1D and 2D NMR methods, 1 and $\mathbf{2}$ were found to be comprised of eight amino acid residues, which were assigned as Gly, two Ser, two Asn, $\beta$-hydroxy-Asn, $\beta$-hydroxy-Tyr, and a new fatty acyl amino acid (FAA; Figure 1). These amino acid

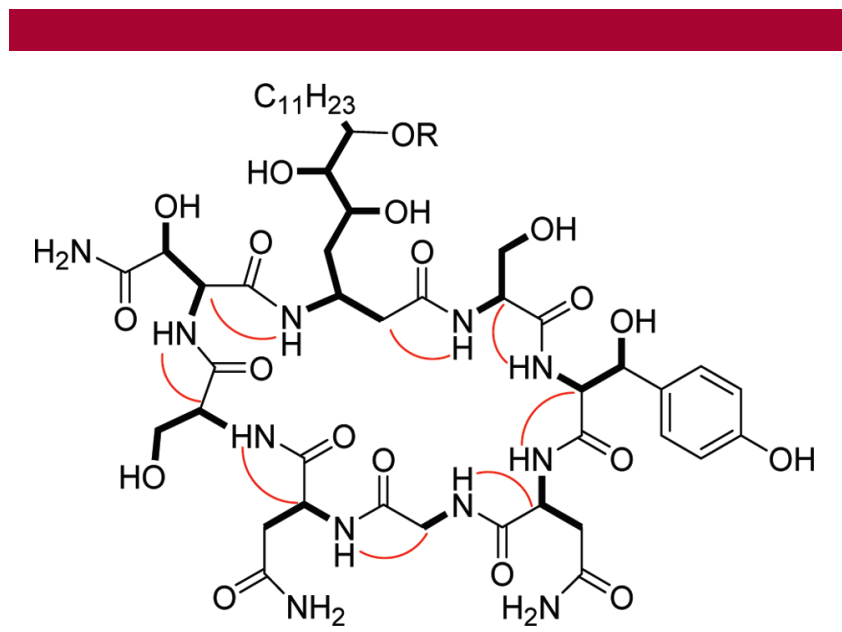

Figure 1. Key COSY (bold black lines) and ROESY (red curved lines) correlations for $\mathbf{1}(\mathrm{R}=$ xylose $)$ and $\mathbf{2}(\mathrm{R}=\mathrm{H})$.

assignments were fully supported by ${ }^{1} \mathrm{H},{ }^{13} \mathrm{C}$, DEPT, COSY, HSQC, HMBC, TOCSY, and ROESY data (Figure 1, Supporting Information, Figures $2-8)$. The ${ }^{1} \mathrm{H}$ NMR spectra revealed the presence of eight main-chain amide protons of $\mathbf{1}$ $(\delta \mathrm{H} 7.32$ to $\delta \mathrm{H} 8.34)$ and $2(\delta \mathrm{H} 7.36$ to $\delta \mathrm{H} \mathrm{8.34)}$ and seven $\mathrm{C} \alpha$ protons in $\mathbf{1}(\delta \mathrm{H} 3.55$ to $\delta \mathrm{H} 4.63)$ and $\mathbf{2}(\delta \mathrm{H} 3.56$ to $\delta \mathrm{H}$ 4.64). An additional set of $\mathrm{C} \alpha$ protons was assigned at 2.30 and 2.41 and corresponded to position 2 of the FAA chain of 1 and 2. Additional amide signals were assigned to the Asn side chains. The resonances for $\beta$-hydroxylated amino acids were identified by the presence of unusual chemical shifts:

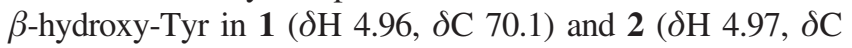

70.3); $\beta$-hydroxy-Asn in $\mathbf{1}(\delta \mathrm{H} 3.96, \delta \mathrm{C} 71.2)$ and $\mathbf{2}(\delta \mathrm{H} 3.96$, $\delta \mathrm{C}$ 71.4). Standard chemical shifts for $\beta$-protons of Ser and Asn were also observed (Supporting Information, Table 1).

Following elucidation of the core amino acids, the remaining signals were assigned to a new FAA moiety (1 and 2 ) and a pentose sugar residue in 1. Five signals between $\delta \mathrm{C} 65.2$ and 101.2 found only in $\mathbf{1}$ comprised the pentose and could be clearly related by COSY, TOCSY, and HMBC. Chemical hydrolysis and HPLC analysis confirmed that this sugar was xylose. A large coupling constant $(J=10.5 \mathrm{~Hz})$ between $\mathrm{H} 1$ and $\mathrm{H} 2$ of xylose established $\mathbf{1}$ as an $\alpha$-xyloside. The remainder of NMR signals could be clearly assigned to FAA, a modified octadecanoic acid derivative. The core of this fatty acid comprises seven carbons with more downfield signals in comparison to unmodified fatty acids. Tellingly, C3 exhibited the correct chemical shifts for an N-linked carbon in $\mathbf{1}(\delta \mathrm{C} 43.6$, $\delta \mathrm{H} 4.20)$ and 2 ( $\delta \mathrm{C} 43.7, \delta \mathrm{H} 4.19)$. This was confirmed by the coupling observed between $\mathrm{H} 3$ and the amide $\mathrm{NH}$ proton of FAA in the COSY spectra. COSY allowed unambiguous assignment of signals from $\mathrm{C} 2-\mathrm{C} 8$, including three unusually downfield signals assigned to hydroxylated $\mathrm{CH}$ groups. $\mathrm{HMBC}$ correlations, particularly from $\mathrm{OH}$ groups, also defined the placement of $\mathrm{C} 2-\mathrm{C} 8$. Finally, a large signal consisting of 18 protons at $\delta \mathrm{H} 1.24$ and $\delta \mathrm{C}$ of $\mathbf{1}(21-35)$ and $\mathbf{2}(21-30)$ was correlated by COSY, TOCSY, and HMBC to a terminal methyl group, $(\delta \mathrm{H} 0.85, \delta \mathrm{C} 13.4)$, allowing complete assignment of FAA (Supporting Information, Figure 9). To the best of our knowledge, this substitution pattern is not found in previously reported fatty acids.

The amino acid sequence of $\mathbf{1}$ and $\mathbf{2}$ was established primarily by ROESY and HMBC correlations (Supporting Information, Figures 6 and 7 and Tables 2 and 3). ROESY correlations between $\alpha$ protons and neighboring residue $\mathrm{NH}$ groups allowed assignment of the order of amino acids (Figure 1). The placement of xylose was confirmed by a series of ROESY and HMBC correlations between xylose and the nearby fatty acyl chain (Supporting Information, Figures 6 and 7 and Tables 2 and 3). MS/MS analysis of $\mathbf{1}$ was complicated by the presence of xylose, which limited fragmentation. By contrast, 2 provided fragment ions that confirmed the NMR-derived sequence (Supporting Information, Figure 1).

Preliminary attempts to establish the absolute configuration of $\mathbf{1}$ and $\mathbf{2}$ employed hydrolysis followed by derivatization with Marfey's reagent. ${ }^{6}$ In comparison to authentic standards, L-threo-OH-Asn was identified; no standard for OH-Tyr is readily available. One equivalent each of D- and L-Ser and D- and L-Asp was found in the hydrolysate (Supporting Information, Figures 12 and 13). To determine the placement of these isomers in $\mathbf{1}$ and $\mathbf{2}$, relatively selective cleavage at Asx using formic acid was performed. ${ }^{7}$ Authentic standards of L-Asp-L-Ser and D-Asp-L-Ser were synthesized. These standards and the formic acid hydrolysate of $\mathbf{1}$ were derivatized with 2,4-dinitro-1-fluorobenzene. ${ }^{8}$ HPLC of the hydrolysate showed the presence of a peak corresponding to either L-Asp-L-Ser or its enantiomer, while no peak for

(6) Marfey, P. Carlsberg Res. Commun. 1984, 49, 591-596.

(7) Li, A.; Sowder, R. C., II; Henderson, L. E.; Moore, Sh. P.; Garfinkel, D. J.; Fisher, R. J. Anal. Chem. 2001, 73, 5395-5402. 
D-Asp-L-Ser or its enantiomer was seen (Supporting Information, Figure 14). To discriminate between the L,L and D,D isomers, the hydrolysate and the standards were derivatized with Marfey's reagent. A peak coeluting with the L,L isomer was observed, indicating that the peptide is D-Ser II, D-Asn I, L-Asn II, L-Ser I (Supporting Information, Figure 15).

The activities of $\mathbf{1}$ and $\mathbf{2}$ were determined in assays against $S$. cerevisiae to be 0.4 and $1.6 \mu \mathrm{g} / \mathrm{mL}$, respectively. These compounds are 16-60-fold more potent than amphotericin control against the same $S$. cerevisiae strain. $\mathbf{1}$ and $\mathbf{2}$ are 2and 16-fold more potent than amphotericin B against A. niger, respectively, and 2 -fold more potent against $C$. albicans (see Supporting Information).

$\mathbf{1}$ and $\mathbf{2}$ are similar to xylocandin B1 and C1, respectively, which contain the same amino acid constituents and a modified FAA. ${ }^{9}$ However, for xylocandins, the modified FAA differs by hydroxylation pattern, and the amino acid sequence and the placement of xylose in xylocandin B1 were not determined (Figure 2). Burkholdines are also similar to
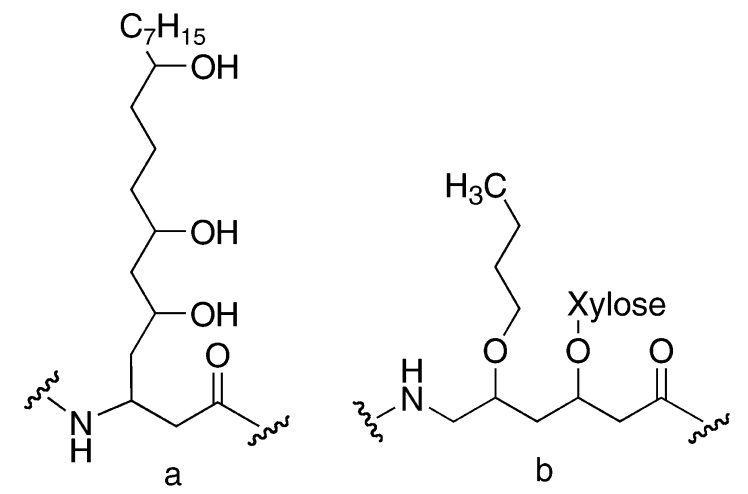

Figure 2. Structures of FAA (as reported) of: (a) xylocandins and (b) 3 .

occidiofungins A and B (3). ${ }^{10} \mathbf{3}$ is identical to $\mathbf{1}$, except that Trp and Lys in $\mathbf{3}$ replace Ser II and Asn I, respectively. There is also a wholly different FAA in $\mathbf{3}$ (Supporting Information, Figures 10 and 11). We propose that $\mathbf{1}$ and $\mathbf{3}$ are actually very similar and that the data should be reexamined (Supporting Information, Table 4 and Figures 9-11). The exact nature of the proposed structure of $\mathbf{3}$ cannot be elucidated from the previously reported data. The original structure assignment of occidiofungins was complicated by an incomplete data set that was obtained using a mixture of compounds, leading to some ambiguity in the data used to assign the original structure. ${ }^{10}$

Burkholdines belong to a family of bioactive, cyclic lipopeptides isolated from $\beta$-proteobacteria such as Burkholderia and Pseudomonas species. ${ }^{9-11}$ Cells and spray dried material from cultures of $B$. ambifaria strain $2.2 \mathrm{~N}$ have shown strong antimicrobial activity against a wide variety of fungal pathogens including Phytophthora infestans, Alternaria solani, Botrytis cinerea, and Mycosphaerella figiensis. ${ }^{4}$ As the likely source of activity against these strains, the burkholdines provide lead structures for development of new antifungal agents.

Acknowledgment. The authors would like to thank Jack Skalicky, University of Utah, for help in obtaining NMR data. This work was supported by the US-Egypt Joint Fund for Science and Technology.

Supporting Information Available: Spectral data for $\mathbf{1}$ and $\mathbf{2}$, detailed methods, and a comparative analysis of $\mathbf{1}$ with $\mathbf{3}$ are provided. This material is available free of charge via the Internet at http://pubs.acs.org.

OL9029269

(8) Song, Y.; Shenwu, M.; Zhao, Sh.; Hou, D.; Liu, Y. J. Chromatogr. A 2005, 1091, 102-109.

(9) Bisacchi, G. S.; Hockstein, R. D.; Koster, W. H.; Parker, W. L.; Rathnum, M. L.; Unger, S. E. J. Antibiot. 1987, 40, 1520-1529.

(10) Lu, S.-E.; Novak, J.; Austin, F. W.; Gu, G.; Ellis, D.; Kirk, M.; Wilson-Stanford, Sh.; Tonelli, M.; Smith, L. Biochemistry 2009, 48, 83128321.

(11) Lim, Y.; Suh, J.-W.; Kim, S.; Hyun, B.; Kim, Ch.; Lee, C.-H. J. Antibiot. 1994, 47, 1406-1416. 